\title{
A non-invasive, automated diagnosis of Menière's disease using radiomics and machine learning on conventional magnetic resonance imaging: A multicentric, case-controlled feasibility study
}

\author{
Marly F. J. A. van der Lubbe ${ }^{1}$ (D . Akshayaa Vaidyanathan ${ }^{2,3} \cdot$ Marjolein de Wit $^{1}$ - Elske L. van den Burg ${ }^{1}$. \\ Alida A. Postma ${ }^{4,5} \cdot$ Tjasse D. Bruintjes $^{6,7}$ - Monique A. L. Bilderbeek-Beckers ${ }^{8}$. Patrick F. M. Dammeijer ${ }^{9}$. \\ Stephanie Vanden Bossche ${ }^{10,11}$. Vincent Van Rompaey ${ }^{12}$. Philippe Lambin ${ }^{2} \cdot$ Marc van Hoof $^{1}$. \\ Raymond van de Berg ${ }^{1}$
}

Received: 2 March 2021 / Accepted: 26 October 2021 / Published online: 25 November 2021

(c) The Author(s) 2021

\begin{abstract}
Purpose This study investigated the feasibility of a new image analysis technique (radiomics) on conventional MRI for the computer-aided diagnosis of Menière's disease.

Materials and methods A retrospective, multicentric diagnostic case-control study was performed. This study included 120 patients with unilateral or bilateral Menière's disease and 140 controls from four centers in the Netherlands and Belgium. Multiple radiomic features were extracted from conventional MRI scans and used to train a machine learning-based, multilayer perceptron classification model to distinguish patients with Menière's disease from controls. The primary outcomes were accuracy, sensitivity, specificity, positive predictive value, and negative predictive value of the classification model.

Results The classification accuracy of the machine learning model on the test set was $82 \%$, with a sensitivity of $83 \%$, and a specificity of $82 \%$. The positive and negative predictive values were $71 \%$, and $90 \%$, respectively.

Conclusion The multi-layer perceptron classification model yielded a precise, high-diagnostic performance in identifying patients with Menière's disease based on radiomic features extracted from conventional T2-weighted MRI scans. In the future, radiomics might serve as a fast and noninvasive decision support system, next to clinical evaluation in the diagnosis of Menière's disease.
\end{abstract}

Keywords Menière's disease $\cdot$ Magnetic resonance imaging $\cdot$ Radiomics $\cdot$ Machine learning

\section{Introduction}

Menière's disease (MD) is a multifactorial condition of the inner ear characterized by recurrent episodes of vertigo and fluctuating aural symptoms like hearing loss, aural fullness, and tinnitus. The exact etiology of the disease is unknown. However, MD is strongly associated with the classical histological finding known as endolymphatic hydrops (EH), which is a distention of the endolymphatic compartment of the labyrinth [1]. The consistent finding of EH in temporal

Marc van Hoof, Raymond van de Berg have equally contributed to do this work

Marly F. J. A. van der Lubbe

marly.lubbe@mumc.nl; marlyvanderlubbe93@gmail.com

Extended author information available on the last page of the article bones of patients with MD [2,3] led to defining EH as the pathological basis of MD. However, it also marked the beginning of a diagnostic challenge, as EH could only be identified post-mortem. As a consequence, MD remained a clinical diagnosis, and different symptom-based classification methods emerged over time [4].

The clinical diagnosis of MD, however, is complicated due to the diverse clinical presentation of the disease, symptom overlap with other etiologies, and the lack of specific biomarkers [5-7]. Therefore, new imaging techniques are under investigation as a MD diagnostic [8,9]. Nowadays, the most commonly applied technique in clinical practice is delayed gadolinium-enhanced MRI $[10,11]$. This technique enables the in-vivo confirmation of EH. Various methods have been proposed to qualitatively and quantitatively assess the endolymphatic space [10]. Most recent developments even allow the fully automatic 3D segmentation and 
volumetric quantification of the endolymphatic space [12, 13], a significant step towards automatization and standardization of EH assessment on imaging. Nevertheless, EH is not a pathognomic feature to MD. It is observed in various neuro-otologic pathologies as well as in asymptomatic ears $[10-12,14]$. The exact relationship between EH and MD and its pathological and clinical relevance are not completely understood. Combining additional image markers with EH, such as the degree of perilymphatic enhancement, seem highly specific for MD $[15,16]$.

The ongoing developments in delayed gadoliniumenhanced MRI are promising for the role of imaging in the diagnosis of MD. However, there are downsides related to contrast-enhanced MRI. It deals with a delayed scanning protocol. Imaging is performed $24 \mathrm{~h}$ after intratympanic (IT) and $4 \mathrm{~h}$ after intravenous (IV) administration of gadolinium [9]. Furthermore, IT administration is considered an invasive procedure $[9,11]$, and IV administration is contraindicated in patients with contrast allergies or renal dysfunction [17]. Although no ototoxicity has been reported [18], adverse effects such as gadolinium deposition in the brain have been observed [17, 19]. Hence, a more efficient, less invasive imaging technique to diagnose MD would be preferable. The concomitant exploration of other potential MD diagnostics remains relevant.

Increasing evidence indicates that diagnostic, prognostic, and predictive information can be extracted from standardof-care image modalities [20-22]. The process of converting medical images into mineable high-dimensional data by extracting quantitative image features and linking them to clinical outcomes is referred to as radiomics [21, 23]. To analyze such large amounts of image features, machine learning (ML) methods are often used to find patterns in the data.

A preliminary study demonstrated the possible value of radiomics within the diagnosis of MD by detecting differences in image features between patients with MD and controls in conventional MRI scans [24]. To further explore the application of radiomics, the objective of this study was to develop a computer-aided diagnostic tool for MD by using a radiomics approach combined with ML. Its performance and feasibility as a new diagnostic tool for MD were evaluated.

\section{Materials and methods}

\section{Study design and inclusion}

A retrospective, diagnostic case-control study was performed on patients with unilateral and bilateral MD. Medical records in the following centers in the Netherlands and Belgium were searched for eligible subjects:
1. Center A (Maastricht University Medical Center +), The Netherlands

2. Center B (Antwerp University Hospital), Belgium

3. Center C (Apeldoorn Dizziness Center), The Netherlands

4. Center D (VieCuri Hospital Venlo), The Netherlands

For the inclusion of subjects, a conventional MRI scan of the cerebellopontine angle already available from the clinical setting was required. Rough motion artifacts and/or unsharp delineation of the inner ear on MRI was an exclusion criterion. Subjects were enrolled as "patients" when clinically diagnosed by an ENT-specialist as definite MD according to the criteria of the American Academy of Otolaryngology-Head and Neck Surgery (AAO-HNS) [25] and/ or Barany society (2015) [4]. Both unilateral and bilateral cases of definite MD were included. Subjects were enrolled as "controls" when diagnosed by an ENT-specialist with idiopathic asymmetric sensorineural hearing loss. The labyrinth least affected by hearing loss was considered the best representative of a 'normal' labyrinth and was included in the study. These patients were chosen as controls since this was a retrospective study and no MRI scans from 'healthy' people without any hearing loss were available. Controls were excluded in case of a documented history of vertigo and/or balance disorders.

\section{Statistical analysis}

A Chi-square test of independence was performed for between-group comparisons of gender distribution and independent samples t-test for age distributions. Statistical analyses were carried out using SPSS software version 25.0 (IBM Corp, Armonk, NY).

\section{Radiomics workflow}

The radiomics workflow applied in this study consisted of four steps, as illustrated in Fig. 1.

\section{MR imaging and segmentation}

Image acquisition and data anonymization were performed by the local investigators of the four centers. T2-weighted MR images were acquired with center-specific protocols on $1.5 \mathrm{~T}$ and $3 \mathrm{~T}$ scanners. Scan parameters were not constant between centers, as shown in Table 1 of the supplementary materials. The 3D Slicer 4.8.1 [26] was used to segment the labyrinth from all MRI scans. Two authors (EB, MW) manually segmented all labyrinths using an inbuilt region-growing algorithm (Grow from seeds) [27]. The first author (ML) cleaned the initial dataset and re-segmented the labyrinths in 


\section{Radiomics workflow}
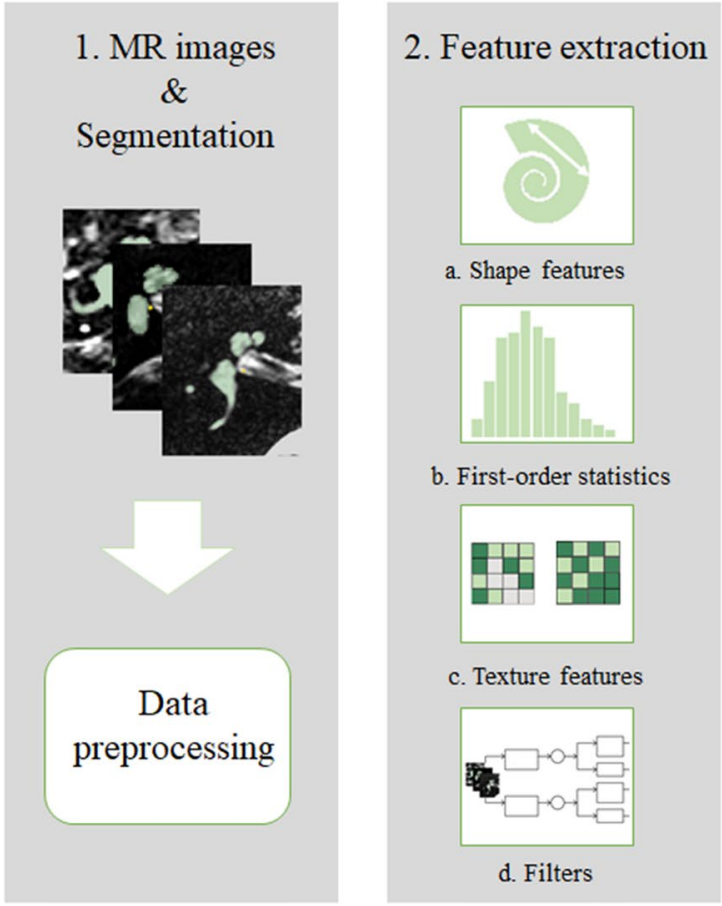

Fig. 1 The workflow of Radiomics in this study is graphically presented in four steps. (1) T2-weighted MR images were collected from four different centers in the Netherlands and Belgium and manually segmented. The MR volumes and their corresponding segmentation masks were preprocessed into isotropic voxels. (2) Four types of fea-

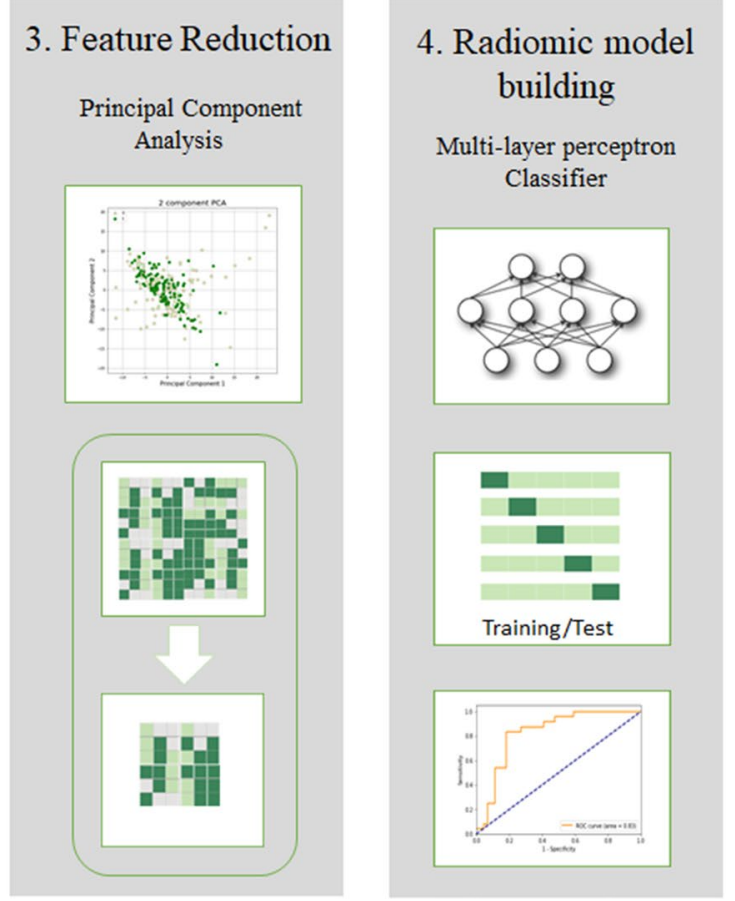

tures (a. Shape features, b. First-order statistic features, c. Texture features, and d. Features extracted after applying different filters) were extracted from the segmented masks. (3) Feature reduction was done by principal component analysis. (4) A multi-layer perceptron classifier was used for radiomic analysis

Table 1 Details of study cohort

\begin{tabular}{|c|c|c|c|c|c|c|c|}
\hline Group & $\mathrm{n}$ & Center & Menière’s (n) & Controls (n) & Age (years) & Gender (M/F) & Date MRI \\
\hline \multirow[t]{4}{*}{ Training cohort (74\%) } & \multirow[t]{4}{*}{192} & A & 40 & 19 & \multirow[t]{4}{*}{$60 \pm 8$} & \multirow[t]{4}{*}{$92 / 67$} & \multirow[t]{4}{*}{ 2004-2017* } \\
\hline & & B & 25 & 20 & & & \\
\hline & & $\mathrm{C}$ & 31 & 57 & & & \\
\hline & & Total & 96 & 96 & & & \\
\hline \multirow[t]{5}{*}{ Test cohort $(26 \%)$} & \multirow[t]{5}{*}{68} & A & 8 & 4 & \multirow[t]{5}{*}{$61 \pm 9$} & \multirow[t]{5}{*}{$34 / 25$} & \multirow[t]{5}{*}{$2004-2017^{*}$} \\
\hline & & B & 8 & 4 & & & \\
\hline & & $\mathrm{C}$ & 2 & 18 & & & \\
\hline & & $\mathrm{D}$ & 6 & 18 & & & \\
\hline & & Total & 24 & 44 & & & \\
\hline
\end{tabular}

Demographic details of the study cohorts. $\mathrm{N}=$ number of ears, Age is median age with median absolute deviation, * Significant difference between cohorts

case of missing labels. Examples of manual segmentations are demonstrated in Fig. 2.

The following preprocessing steps were performed [28]. 1) in order to normalize the voxel sizes across the volumes, the MR volumes and their corresponding segmentation masks were resampled to isotropic voxels of length $0.5 \mathrm{~mm}$ using cubic spline interpolation. 2) voxel intensities were transformed using $\mathrm{Z}$-score normalization to minimize the influence of contrast or brightness variation among the images. 3) the transformed voxel intensities were discretized using a fixed bin width of 0.5 . 
Fig. 2 a A cropped MR image of a right inner ear of a subject with asymmetric sensorineural hearing loss on the right side. From left to right, the axial, sagittal and coronal planes are presented. The manual segmentation is visualized by the green mask $\mathbf{b}$ A cropped MR image of a left inner ear of a subject with unilateral Menière's disease on the left side. From left to right, the axial, sagittal and coronal planes are presented. The manual segmentation is visualized by the green mask a

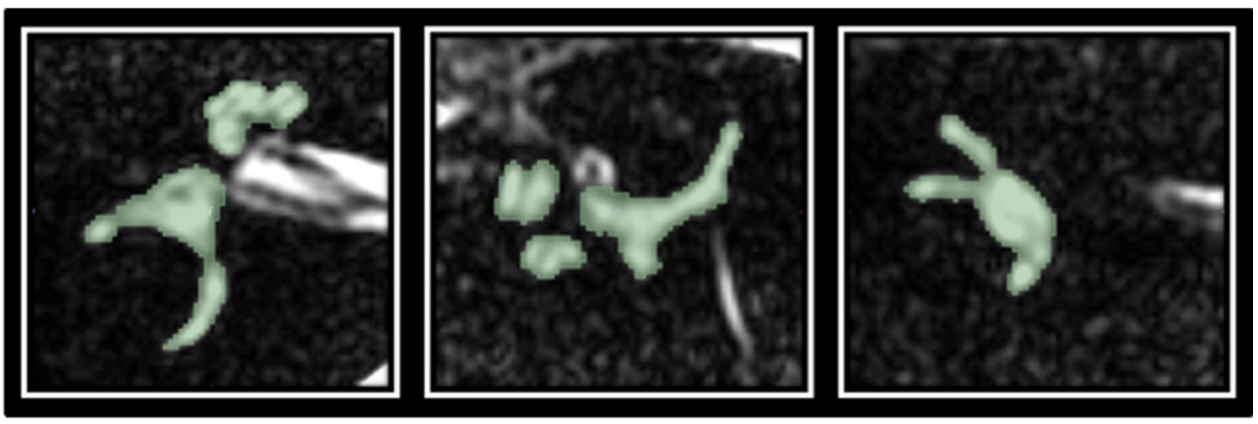

b

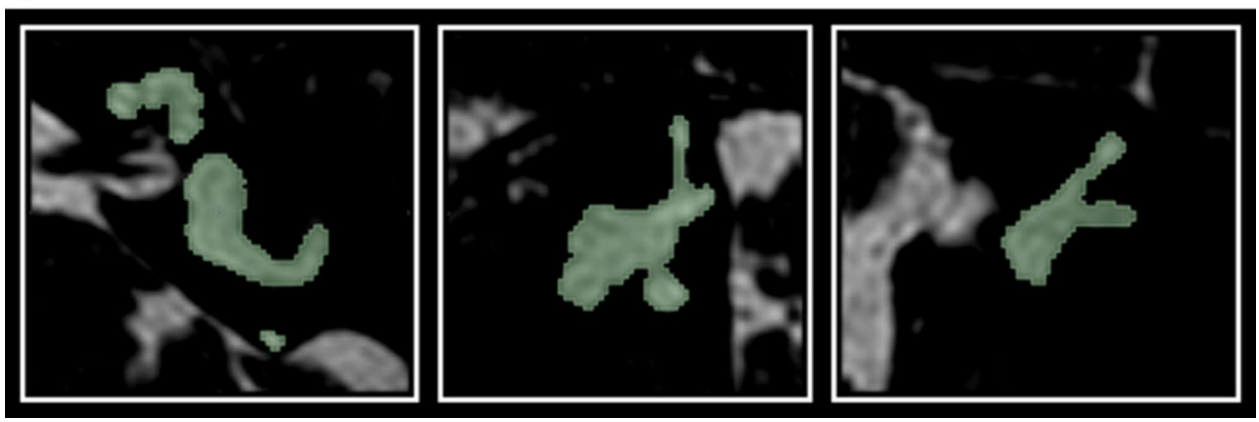

\section{Feature extraction}

In total, 812 radiomic features were extracted from the segmented masks using RadiomX (Oncoradiomics SA, Liège, Belgium). Mathematical descriptions of all features were previously published and presented as Supplemental Material with the permission of the corresponding authors [20, 22, 29].

\section{Feature reduction}

To reduce the dimensionality of the extracted features, a principal component analysis (PCA) was performed. PCA is an unsupervised, linear dimensionality reduction technique in which small numbers of uncorrelated variables are extracted as "Principal Components" to explain most of the variation in the data in lower dimensions [30, 31]. As a result, essential information holding most of the variation in the data was preserved, and non-essential parts with fewer variations were removed. Ten Principal Components were extracted from the analysis and used to train the model. The inverse PCA was applied to identify the mean contribution of each feature overall principal components in order to predict the most important features. A mean contribution of $>0.7$ was chosen to identify 15 features, which had the largest contribution to the PCA.

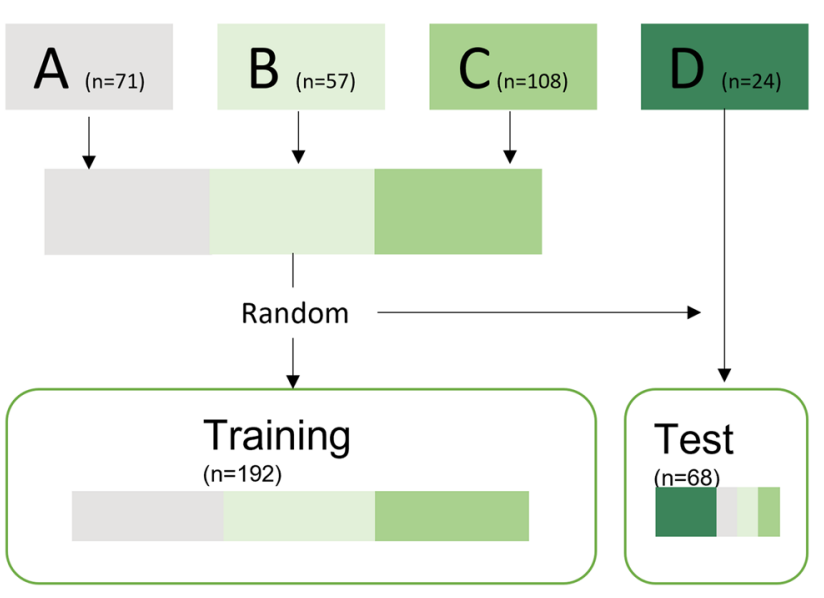

Fig. 3 The flowchart of the train-test data split

\section{Radiomic model building}

A Multi-Layer Perceptron classifier with 500 units in the hidden layer with Adam optimizer at a learning rate of 0.001 was selected for the classification task. The input to the model was the extracted Principal Components. The output layer consisted of a single neuron for each prediction class (patients $=1$ and control=0), which used the Softmax function to output a value between 0 and 1 . The output represented the probability of the predicted classes. The regularization method "early stopping" was adopted during training to avoid overfitting the model [32]. 
Two methods were used for the evaluation of the ML model: a train-test split and k-fold cross-validation.

Train-test split: The complete dataset ("patients" and "controls") was divided into a training and test set of $74 \%$ and $26 \%$, respectively. The training set contained images from centers $\mathrm{A}, \mathrm{B}$ and $\mathrm{C}$. The test set contained images from center D, as an external center. The test set was complemented with randomly selected scans from the other centers (A, B, and C), which were excluded from training. This means no cases were used in both training and testing. The train-test split is graphically presented in Fig. 3.

$K$-fold cross-validation: A 10-fold cross-validation was performed on the complete dataset. The data were randomly split into ten parts. Alternately, nine parts were used for training and one part for testing.

\section{Outcome measurements}

The primary outcomes of this study were accuracy, sensitivity, specificity, positive predictive value, and negative predictive value. The precision (i.e., confidence interval) of each parameter was determined. As additional outcomes, the F1-scores and the Matthews correlation coefficients were reported.

\section{Results}

\section{Study population}

This retrospective study included 120 patients with MD (59 men, 61 women, aged 16-84; median age 59, median absolute difference \pm 9$)$ and 140 controls with asymmetric sensorineural hearing loss (67 men, 31 women in 42 controls gender was unknown, aged 6-88; median age 63, median absolute difference \pm 7 ) over four centers. There were 71 labyrinths included from Center A $(67.6 \% \mathrm{MD}, 32.4 \%$ control), 57 from Center B (59.1\% MD, 42.1\% control), 108 from Center C (30.6\% MD, 69.4\% control), and 24 from Center D (35.3\% MD, 64.7\% control) There was no significant difference in age distribution between the patient and the control group and between the training and test cohort. The proportion of known males versus females did not differ between the test and training cohort. A significant difference in scan date between the training and test cohort was found (independent sample t-test: $p=0.019$ ) with MRI scans of the training cohort being performed on earlier dates. No significant differences in scan date between all patients with MD and controls were found. Details of the training and test cohort are presented in Table 1.

\section{Principal component analysis}

By applying the inverse PCA, the mean contribution of each feature over all principal components is illustrated in Fig. 4. As a result, the features with the most substantial influence on the principal components could be identified. These are presented in Table 2 of the Supplementary Materials.

\section{Machine learning classifier}

The ML model's performance on classifying patients with MD and controls is demonstrated in Table 2. The
Fig. 4 The mean contribution over all principal components aggregated for each feature. The red line indicates the cutoff value $(<0.7)$ for the most important features that contributed to the PCA

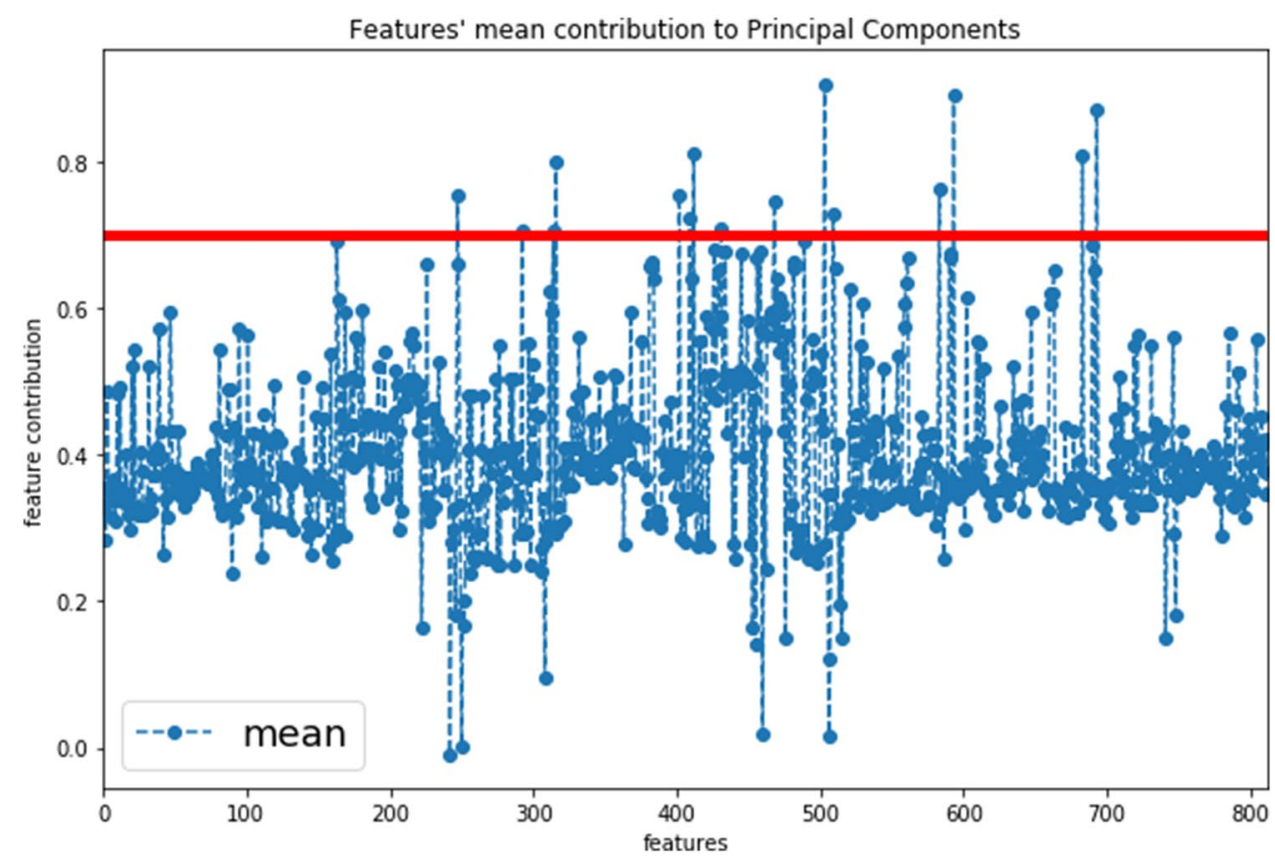


Table 2 Classification performance

\begin{tabular}{llll}
\hline & Training cohort & Test cohort & $\begin{array}{l}\text { 10-fold cross-valida- } \\
\text { tion }\end{array}$ \\
\hline Patients vs. Controls & 96 vs. 96 & 24 vs. 44 & \\
Accuracy (\%) & 72.9 & 82.3 & 80.0 \\
AUC (95\% CI) & $80.6(80.5-81.2)$ & $86.9(86.6-88.8)$ & $83.6(77.9-89.3)$ \\
Sensitivity (95\% CI) & $80.2(80.0-81.1)$ & $83.4(82.6-86.9)$ & $78.3(71.4-85.3)$ \\
Specificity (95\% CI) & $65.6(65.3-66.3)$ & $81.8(81.4-83.7)$ & $77.5(70.5-84.5)$ \\
Positive predictive value (95\% CI) & $70.0(69.7-70.6)$ & $71.4(70.4-74.1)$ & $77.6(69.9-85.4)$ \\
Negative predictive value (95\% CI) & $76.8(67.5-77.8)$ & $90.0(89.7-92.3)$ & $78.4(70.6-86.3)$ \\
F1-scores & 0.75 & 0.77 & 0.77 \\
MCC & 0.46 & 0.63 & 0.56 \\
\hline
\end{tabular}

Performance of the multi-layer perceptron classification metric to distinguish MD from healthy controls showing the area under the curve of the Receiver Operating Curve, sensitivity, specificity, positive predictive value, negative predictive value, F1-scores and MCC. The mean F1-scores, and MCC of the 10-fold cross-validation are presented. Abbreviations: $C I$ Confidence interval, $A U C$ Area under the curve, $M C C$ Matthews correlation coefficient

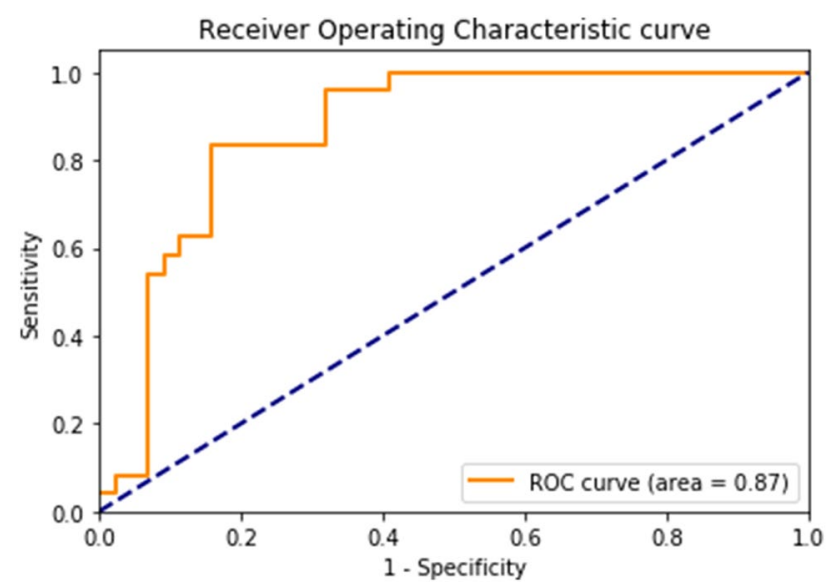

Fig. 5 The Receiver Operator Characteristic curve of the test cohort of the multi-layer perceptron classifier

classification accuracy of the test set was $82 \%$, with a sensitivity of $83 \%$, specificity of $82 \%$, and AUC of $87 \%$. The positive and negative predictive values were $71 \%$ and $90 \%$, respectively. The ROC curve and the confusion matrix are shown in Figs. 5 and 6. The F1-scores and the Matthews correlation coefficients were 0.75 and 0.46 for training and 0.77 and 0.63 for testing, respectively. The results of the 10 -fold cross-validation are also presented in Table 2. The mean classification accuracy across the 10-fold was $80 \%$, with a mean sensitivity, specificity, positive predictive value, and negative predictive value of $78 \%, 77.5 \%, 78 \%$, and $78 \%$, respectively. The mean AUC was $84 \%$.

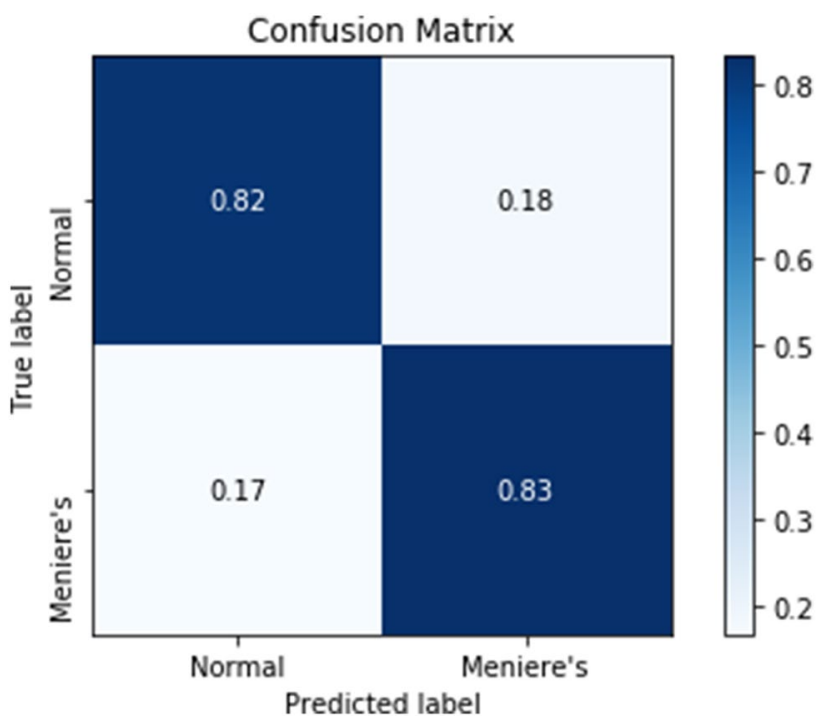

Fig. 6 The confusion matrix of the test cohort of the multi-layer perceptron classifier. The true labels are the diagnostic labels after subject inclusion. The predicted labels are the labels predicted by the classifier

\section{Discussion}

This study demonstrated that radiomic features extracted from conventional MRIs could be used to discriminate MD patients from 'normal' controls with high accuracy. In a heterogenic, multicentric dataset, the proposed ML model yielded a precise, high-diagnostic performance in identifying patients with MD with an accuracy of $82 \%$. The results are in line with the preliminary study [24], where the feasibility of radiomics was first explored in a small, single-center setup. By integrating ML, the present study proved the value of radiomics in a larger dataset containing heterogeneous 
images from multiple centers and reported the performance of a diagnostic classification model for MD. The next goal would be to investigate the feasibility of a clinical, prospective application.

\section{Relevance of radiomics in the diagnosis of MD}

Within neuro-otology, radiomics is a very new concept. This study pioneered in developing a computer-aided diagnostic tool for MD by using a radiomic approach. Research on the role of imaging within MD's diagnosis mainly focuses on the in-vivo visualization of $\mathrm{EH}$ on delayed gadoliniumenhanced MRI. One of the main benefits of radiomics is that it can also be applied to MRI scans acquired with no contrast agent. Within the diagnostic workup of MD, these scans are often readily available since MRI is indicated to exclude other causes of asymmetrical hearing loss. Radiomics is not just reserved for centers with specialized radiological expertise on EH. Instead, it could be performed in any center with a (1.5 T or $3 \mathrm{~T}) \mathrm{MRI}$ scanner, without a delayed scanning protocol, and also in case of allergic reaction to contrast media.

Other studies have also investigated the feasibility of the radiological diagnosis of MD without the use of contrast agents. One publication showed changes in the membranous labyrinth between patients with MD and healthy subjects on 3D CBCT and suggested the usefulness of 3D CBCT imaging for the objective diagnosis of MD [8]. However, the diagnostic value has not been clinically evaluated yet. Three other publications proposed the use of non-contrast T2-weighted MRI for the diagnosis of MD by manually measuring the length and width of the saccule and/or the utricle [33-35]. One of these papers reported the maximum saccular height in healthy volunteers to be $1.6 \mathrm{~mm}$ [33]. Another reported a high specificity (95\%) but a low sensitivity (63\%) for a cut-off value of $1.51 \mathrm{~mm}$ for saccular height [34]. Measurements of the absolute utricle area and the utricle-to-vestibule area ratio were also identified as predictors of MD and yielded a sensitivity of $44 \%$ and $75 \%$ and a specificity of $81 \%$ and $53 \%$, respectively. The main disadvantage of these techniques is that only the vestibule and the basal turn of the cochlea were evaluated, while the radiomics method assessed the entire labyrinth. Moreover, human performances on non-contrast MR imaging seem to exhibit lower diagnostic performance compared to radiomics. Prospective studies performing radiomics and vestibular measurements on the same dataset are needed to prove this assumption.

Another benefit of radiomics is that it has the potential to serve as a fully automated diagnostic tool for MD. This study still extracted radiomic features from manually segmented inner ears. Manual segmentation is time-consuming and prone to intra- and inter-observer variability and, therefore, difficult to transpose to routine radiology [36, 37]. However, automatic segmentation of the inner ear has already been made accessible [38]. When integrated with the automatic segmentation of the inner ear, radiomics could be used as a standardized decision support system that might reduce human interference and, therefore, interobserver variability within and between centers. Further research is necessary to evaluate the model's performance on features extracted from automatically segmented labyrinths.

Eventually, it would be interesting to compare the diagnostic values of automated radiomic analysis with the automatic volumetric assessment of EH on contrast-enhanced MRI.

\section{Interpretations and mechanism}

An essential hypothesis of radiomics is that underlying tissue heterogeneity, associated with cellular and molecular biology, could be captured by the quantitative features extracted from medical images [20, 39]. For instance, several studies have shown a relationship between radiomic features and gene-expression patterns in patients with cancer [22, 40].

The predictive power of the multi-layer perceptron classifier suggested that the extracted radiomic features captured differences in the labyrinth of patients with MD and controls. Hypothetically, these differences could mirror changes in the morphology or perhaps even changes in the molecular biology of the inner ear of patients with MD, allowing an improved understanding of the pathophysiology of the disease. It would be valuable to look into the most discriminative features and identify them as visually perceptible or even visually non-perceptual image biomarkers for MD.

However, one of the main drawbacks of machine learning is the interpretability of the underlying mechanisms on which an ML algorithm generates its output, which is why these methods are often referred to as a "black box" [41]. This study attempted to provide some insights into the "rationale" behind the ML model by identifying the extracted features that contributed most to the principal components and thus, in turn, contributed to a highly predictive model.

An interesting finding was that all of these features were extracted after applying a discrete, one-level three-dimensional wavelet-transform to each MRI [20, 22, 42]. Wavelet-transform effectively decouples textural information by decomposing the original image in low and high frequencies $[20,22,42]$. Wavelet decompositions, however, are mathematically generated. It is not yet possible to explain this mathematical information in a clinical manner [43]. Notwithstanding, it was striking that particularly "intensity" features were identified as the most important features. Lower-signal regions on high-resolution T2-weighted MR images have been previously used as margins for saccular 
size and morphology, which seems predictive for MD [34, 35]. One could hypothesize that features such as the "minimum gray level" (Stats_min) or the " $10^{\text {th }}$ percentile of gray level" (Stats_p10) reflected these lower-signal regions and might be linked to saccular hydrops. However, to date, there are no tools to prove this assumption. It remains unclear which (semantic) features would serve as relevant image markers for MD.

\section{Limitations}

This study has several limitations. Firstly, it is important to recognize that no gold standard test is available to compare the radiomics method with. This retrospective study included patients clinically diagnosed with definite MD according to the AAO-HNS criteria [4]. These patients, however, do not represent the full clinical spectrum. After all, patients who do not fulfill these criteria due to the fluctuating aspect of hearing loss (not captured by audiometry) or atypical symptom presentation might be an interesting group to explore with radiomics.

Secondly, the duration and severity of the disease were not considered in this study cohort. Disease duration might alter the morphology of the labyrinth. For example, the severity of EH in patients with MD seems to increase with the duration of the disease $[44,45]$. Perhaps, the disease duration could alter the composition of the endolymphatic fluid as well. The patient cohort in this study probably contained patients with different disease stages. Early disease stages might be challenging to recognize since important image features were not yet significantly present. Adding clinical information about disease duration would probably have improved the model's performance.

Thirdly, the proposed radiomics analysis relied on manual segmentation, which is a limitation to overcome in future studies. Inter- and intra-observer variability in manual segmentation was assumed but not assessed in the present study. Although the vestibular aqueduct could contain valuable information for the diagnosis of MD [46], this structure was not specifically considered in the study due to difficulties in manual segmentation. All are relevant points to keep in mind for future studies.

Fourthly, the study dealt with a small dataset consisting of MRI scans from four independent centers. In the absence of sufficient datapoints, it was inevitable to divide the data into just a training and test set, where ideally, a third split would be made to provide an unbiased evaluation of the model. Cross-validation was adopted to help reduce biased results [47]. In addition, this study included an independent dataset (Center D) in testing to better detect overfitting. Overfitting happens when the model learns details and noise to fit the training data with high accuracy but fails to perform on a new set of data $[32,48]$. The addition of an external set in testing helps to apply early stopping when the model starts to overfit on the training dataset (i.e., when the training loss decreases and validation loss starts to increase). Due to the small size of the training dataset, overfitting could not be completely avoided. The risk of overfitting was further contained by diversifying the training data. This was done by acquiring data from four different centers and by manually segmenting the labyrinth by three different observers. Therefore, the model should be more generalizable for differences in center-specific scan parameters and inter-reader segmentations.

Lastly, the heterogeneities in voxel spacing and slice thickness between the images were handled by isotropic resampling. This could have induced noise due to interpolations. The results of this study are encouraging as a proofof-concept. Additional studies with more training data and validation on multiple external datasets with images from different MRI scanners are needed before definitively claiming the model's generalizability. Exploring the use of convolutional neural networks for the direct extraction of deep features from the raw MRI [49] might also improve the diagnostic accuracy and the generalizability of the model.

\section{Clinical implications and future perspectives}

Radiomics is a new imaging analysis technique that could enable standardized, non-invasive, and widely accessible diagnostic care for patients with MD. The output of the multi-layer perceptron classifier provides a value between 0 and 1 , which represents the probability of the predicted classes. This will allow clinicians to interpret the probability of having MD based on the features extracted from MRIs together with the clinical profile of the patients. Before clinical implementation, further research should focus on three main aspects: 1) Integrating auto-segmentation of the inner ear with radiomics analyses, 2) Further validation of the classification model on a larger training dataset and external validation on multiple external datasets, and 3) Evaluation of the diagnostic value of radiomics as an equivalent of contrast-enhanced MR imaging.

The potential role of radiomics, for now, is mainly to aid the clinical diagnosis of MD as a clinical decision support system. However, there lie more perspectives in the future for radiomics. In the current study, only patients with MD were included. However, radiomics might apply to other labyrinthine disorders as well. One study indicated that cochlea CT image features could be useful biomarkers for predicting sensorineural hearing loss in patients treated with chemoradiotherapy for head and neck cancer [50]. It would be valuable to study the relationship between radiomic features and hearing loss in different causes of sensorineural hearing loss. Performing radiomics in more patients with different disorders will eventually allow a comparison between the general 
vestibular population and a healthy population. Performing a cluster analysis might reveal a reclassification of vestibular disorders based on similarities in radiomic signatures. Compared to symptoms, radiomic signatures might better classify vestibular disorders.

\section{Conclusion}

The automated extraction of radiomic features from conventional MRI scans proved to be valuable to discriminate patients with Menière's disease and 'normal' controls. In the current study, the machine learning-based multi-layer perceptron network yielded a precise, high-diagnostic performance in identifying patients with Menière's disease with an accuracy of $82 \%$. In the future, radiomics could possibly be implemented as a fast, non-invasive, and accurate decision support system, next to clinical evaluation, in the diagnostic trajectory of Menière's disease.

Supplementary Information The online version contains supplementary material available at https://doi.org/10.1007/s11547-021-01425-w.

Acknowledgments Dr. Philippe Lambin reports, inside the submitted work, grants/sponsored research from Oncoradiomics, ptTheragnostic/ DNAmito; advisor (SAB)/presenter fee from Oncoradiomics, Varian medical and Elekta. Dr. Lambin is the inventor of two patents on radiomics and one non-patentable invention (software), licensed to Oncoradiomics and has (minority) shares in the company Oncoradiomics. Akshayaa Vaidyanathan is an employee at Oncoradiomics SA in the framework of a Marie Curie Training Network coordinated by PL. Dr. Vincent Van Rompaey reports on unrestricted research and travel grants paid to the institution by Medel and Cochlear, unrelated to the work.

Author contributions MH, RB Conceptualization, MH, AV Methodology, Software, MFJAL, AV Validation, Formal analysis, Visualization, Writing original draft, MFJAL, MW, ELB Investigation, AAP, TDB, MALB, PFMD, VR, SVB Resources, RB, MH, PL Supervision, PL, MB, VR Funding acquisition, MW, ELB, AAP, TDB, MALB, PFMD, VR, MH, PL, RB Writing - Review \& Editing.

Funding The authors acknowledge financial support from MUMC + and VieCurie Venlo, ERC advanced grant (ERC-ADG-2015, $\mathrm{n}^{\circ}$ 694812) - Hypoximmuno and the European Program H2020 (PREDICT-ITN $-\mathrm{n}^{\circ}$ 766276), Interreg V-A Euregio Meuse-Rhine ("Euradiomics").

\section{Declarations}

Conflict of interest The authors declare that they have no conflict of interest.

Ethics approval This study was performed in accordance with the guidelines outlined by Dutch and Belgium legislation. Subjects were enrolled and fully anonymized by the local investigators (Maastricht University Medical Center, University Hospital Antwerp, VieCuri Hospital Venlo, and Apeldoorn Dizziness Center) and were therefore not asked for their consent. According to the Medical Research Involv- ing Human Subjects Act (WMO), ethical approval was not required due to the retrospective nature and anonymization of the data.

Open Access This article is licensed under a Creative Commons Attribution 4.0 International License, which permits use, sharing, adaptation, distribution and reproduction in any medium or format, as long as you give appropriate credit to the original author(s) and the source, provide a link to the Creative Commons licence, and indicate if changes were made. The images or other third party material in this article are included in the article's Creative Commons licence, unless indicated otherwise in a credit line to the material. If material is not included in the article's Creative Commons licence and your intended use is not permitted by statutory regulation or exceeds the permitted use, you will need to obtain permission directly from the copyright holder. To view a copy of this licence, visit http://creativecommons.org/licenses/by/4.0/.

\section{References}

1. Cairns H (1980) Observations on the pathology of Meniere's syndrome. J Laryngol Otol. https://doi.org/10.1017/S002221510 008960X

2. Merchant SN, Adams JC, Nadol JB (2005) Pathophysiology of Ménière's syndrome: Are symptoms caused by endolymphatic hydrops? Otol Neurotol 26(1):74-81

3. Foster CA, Breeze RE (2013) Endolymphatic hydrops in Ménière's disease: cause, consequence, or epiphenomenon? Otol Neurotol. https://doi.org/10.1097/MAO.0b013e31829e83df

4. Lopez-Escamez JA, Carey J, Chung WH et al (2015) Diagnostic criteria for Menière's disease. J Vestib Res Equilib Orientat. https://doi.org/10.3233/VES-150549

5. Pyykkö I, Nakashima T, Yoshida T et al (2013) Ménière's disease: A reappraisal supported by a variable latency of symptoms and the MRI visualisation of endolymphatic hydrops. BMJ Open. https:// doi.org/10.1136/bmjopen-2012-001555

6. Vassiliou A, Vlastarakos PV, Maragoudakis P et al (2011) Meniere's disease: still a mystery disease with difficult differential diagnosis. Ann Indian Acad Neurol. https://doi.org/10.4103/ 0972-2327.78043

7. Lopez-Escamez JA, Dlugaiczyk J, Jacobs J et al (2014) Accompanying symptoms overlap during attacks in Menière's disease and vestibular migraine. Front Neurol. https://doi.org/10.3389/fneur. 2014.00265

8. Yamane H, Iguchi H, Konishi K et al (2014) Three-dimensional cone beam computed tomography imaging of the membranous labyrinth in patients with Meniere's disease. Acta Otolaryngol. https://doi.org/10.3109/00016489.2014.913315

9. Naganawa S, Nakashima T (2014) Visualization of endolymphatic hydrops with MR imaging in patients with Ménière's disease and related pathologies: Current status of its methods and clinical significance. Jpn J Radiol 32(4):191-204

10. Boegle R, Gerb J, Kierig E et al (2021) Intravenous delayed gadolinium-enhanced $\mathrm{mr}$ imaging of the endolymphatic space: a methodological comparative study. Front Neurol. https://doi.org/ 10.3389/fneur.2021.647296

11. de Pont LMH, van Steekelenburg JM, Verbist BM et al (2020) State of the art imaging in menière's disease tips and tricks for protocol and interpretation. Curr Radiol Rep 8(11):1-4

12. Gerb J, Ahmadi SA, Kierig E et al (2020) VOLT: a novel opensource pipeline for automatic segmentation of endolymphatic space in inner ear MRI. J Neurol. https://doi.org/10.1007/ s00415-020-10062-8 
13. Cho YS, Cho K, Park CJ et al (2020) Automated measurement of hydrops ratio from MRI in patients with Ménière's disease using CNN-based segmentation. Sci Rep. https://doi.org/10.1038/ s41598-020-63887-8

14. van der Lubbe MFJA, Vaidyanathan A, Van Rompaey V et al (2020) The "hype" of hydrops in classifying vestibular disorders: a narrative review. J Neurol 267:197-211

15. Bernaerts A, Vanspauwen R, Blaivie C et al (2019) The value of four stage vestibular hydrops grading and asymmetric perilymphatic enhancement in the diagnosis of Menière's disease on MRI. Neuroradiology. https://doi.org/10.1007/s00234-019-02155-7

16. Van Steekelenburg JM, Van Weijnen A, De Pont LMH et al (2020) Value of endolymphatic hydrops and perilymph signal intensity in suspected Ménière disease. Am J Neuroradiol. https://doi.org/10. 3174/AJNR.A6410

17 Rose TA, Choi JW (2015) Intravenous imaging contrast media complications: the basics that every clinician needs to know. Am J Med 128(9):943-949

18. Louza J, Krause E, Gürkov R (2015) Hearing function after intratympanic application of gadolinium-based contrast agent: a long-term evaluation. Laryngoscope. https://doi.org/10.1002/lary. 25259

19 Gulani V, Calamante F, Shellock FG et al (2017) Gadolinium deposition in the brain: summary of evidence and recommendations. Lancet Neurol 16(7):564-570

20. Lambin P, Rios-Velazquez E, Leijenaar R et al (2012) Radiomics: Extracting more information from medical images using advanced feature analysis. Eur J Cancer. https://doi.org/10.1016/j.ejca.2011. 11.036

21 Gillies RJ, Kinahan PE, Hricak H (2016) Radiomics: images are more than pictures, they are data. Radiology. https://doi.org/10.1148/ radiol.2015151169

22. Aerts HJWL, Velazquez ER, Leijenaar RTH et al (2014) Decoding tumour phenotype by noninvasive imaging using a quantitative radiomics approach. Nat Commun. https://doi.org/10.1038/ncomm s5006

23. Cho YS, Choi SH, Park KH et al (2010) Prevalence of otolaryngologic diseases in South Korea: data from the Korea national health and nutrition examination survey 2008. Clin Exp Otorhinolaryngol. https://doi.org/10.3342/ceo.2010.3.4.183

24. van den Burg EL, van Hoof M, Postma AA et al (2016) An exploratory study to detect ménière's disease in conventional MRI scans using radiomics. Front Neurol. https://doi.org/10.3389/fneur.2016. 00190

25. Adams JC (1995) Committee on Hearing and Equilibrium guidelines for the diagnosis and evaluation of therapy in Meniere's disease. Otolaryngol-Head Neck Surg. https://doi.org/10.1016/S01945998(95)70102-8

26. Fedorov A, Beichel R, Kalpathy-Cramer J et al (2012) 3D Slicer as an image computing platform for the Quantitative Imaging Network. Magn Reson Imaging. https://doi.org/10.1016/j.mri.2012.05.001

27. Egger J, Kapur T, Fedorov A et al (2013) GBM volumetry using the 3D slicer medical image computing platform. Sci Rep. https://doi. org/10.1038/srep01364

28. Carré A, Klausner G, Edjlali M et al (2020) Standardization of brain MR images across machines and protocols: bridging the gap for MRI-based radiomics. Sci Rep. https://doi.org/10.1038/ s41598-020-69298-Z

29. van Timmeren JE, Leijenaar RTH, van Elmpt W et al (2017) Feature selection methodology for longitudinal cone-beam CT radiomics.
Acta Oncol (Madr). https://doi.org/10.1080/0284186X.2017.13502 85

30. Song F, Guo Z, Mei D (2010) Feature selection using principal component analysis. In: Proceedings - 2010 International Conference on System Science, Engineering Design and Manufacturing Informatization, ICSEM 2010

31. Hira ZM, Gillies DF (2015) A review of feature selection and feature extraction methods applied on microarray data. Adv Bioinformatics. https://doi.org/10.1155/2015/198363

32. Caruana R, Lawrence S, Giles L (2001) Overfitting in neural nets: Backpropagation, conjugate gradient, and early stopping. In: Advances in Neural Information Processing Systems

33. Venkatasamy A, Veillon F, Fleury A et al (2017) Imaging of the saccule for the diagnosis of endolymphatic hydrops in Meniere disease, using a three-dimensional T2-weighted steady state free precession sequence: accurate, fast, and without contrast material intravenous injection. Eur Radiol Exp. https://doi.org/10.1186/ s41747-017-0020-7

34. Simon F, Guichard JP, Kania R et al (2017) Saccular measurements in routine MRI can predict hydrops in Menière's disease. Eur Arch Oto-Rhino-Laryngology. https://doi.org/10.1007/ s00405-017-4756-8

35. Keller JH, Hirsch BE, Marovich RS, Branstetter BF (2017) Detection of endolymphatic hydrops using traditional MR imaging sequences. Am J Otolaryngol - Head Neck Med Surg. https://doi. org/10.1016/j.amjoto.2017.01.038

36 Akkus Z, Galimzianova A, Hoogi A et al (2017) Deep learning for brain mri segmentation: state of the art and future directions. J Digit Imaging 30(4):449-459

37. Nogovitsyn N, Souza R, Muller M et al (2019) Testing a deep convolutional neural network for automated hippocampus segmentation in a longitudinal sample of healthy participants. Neuroimage. https:// doi.org/10.1016/j.neuroimage.2019.05.017

38. Vaidyanathan A, van der Lubbe MFJA, Leijenaar RTH et al (2021) Deep learning for the fully automated segmentation of the inner ear on MRI. Sci Rep. https://doi.org/10.1038/s41598-021-82289-y

39. Morin O, Vallières M, Jochems A et al (2018) A deep look into the future of quantitative imaging in oncology: a statement of working principles and proposal for change. Int J Radiat Oncol Biol Phys 102(4):1074-1082

40 Lambin P, Leijenaar RTH, Deist TM et al (2017) Radiomics: the bridge between medical imaging and personalized medicine. Nat Rev Clin Oncol 14(12):749-762

41. Sajda P (2006) Machine learning for detection and diagnosis of disease. Annu Rev Biomed Eng. https://doi.org/10.1146/annurev. bioeng.8.061505.095802

42. van Timmeren JE, Leijenaar RT, van Elmpt W, Reymen B, Lambin $P$ (2017) Feature selection methodology for longitudinal cone-beam CT radiomics. Acta Oncol Madr. https://doi.org/10.1080/0284186X. 2017.1350285

43. Wang S, Yang M, Du S et al (2016) Wavelet entropy and directed acyclic graph support vector machine for detection of patients with unilateral hearing loss in MRI scanning. Front Comput Neurosci. https://doi.org/10.3389/fncom.2016.00106

44. Fukushima M, Kitahara T, Oya R et al (2017) Longitudinal upregulation of endolymphatic hydrops in patients with Meniere's disease during medical treatment. Laryngoscope Investig Otolaryngol. https://doi.org/10.1002/lio2.115

45. Fiorino F, Pizzini FB, Beltramello A, Barbieri F (2011) Progression of endolymphatic hydrops in ménière's disease as evaluated by 
magnetic resonance imaging. Otol Neurotol. https://doi.org/10.1097/ MAO.0b013e31822a1ce2

46. Attyé A, Barma M, Schmerber S et al (2018) The vestibular aqueduct sign: Magnetic resonance imaging can detect abnormalities in both ears of patients with unilateral Meniere's disease. J Neuroradiol. https://doi.org/10.1016/j.neurad.2018.10.003

47. Rafało M (2021) Cross validation methods: analysis based on diagnostics of thyroid cancer metastasis. ICT Express. https://doi.org/ 10.1016/j.icte.2021.05.001

48. Geras KJ (2018) Exploiting diversity for efficient machine learning

49. Yun J, Park JE, Lee H et al (2019) Radiomic features and multilayer perceptron network classifier: a robust MRI classification strategy for distinguishing glioblastoma from primary central nervous system lymphoma. Sci Rep. https://doi.org/10.1038/s41598-019-42276-w

50. Abdollahi H, Mostafaei S, Cheraghi S et al (2018) Cochlea CT radiomics predicts chemoradiotherapy induced sensorineural hearing loss in head and neck cancer patients: a machine learning and multivariable modelling study. Phys Medica. https://doi.org/10.1016/j. ejmp.2017.10.008

Publisher's Note Springer Nature remains neutral with regard to jurisdictional claims in published maps and institutional affiliations.

\section{Authors and Affiliations}

\section{Marly F. J. A. van der Lubbe ${ }^{1}$ (1) - Akshayaa Vaidyanathan ${ }^{2,3} \cdot$ Marjolein de Wit $^{1}$ - Elske L. van den Burg ${ }^{1}$. Alida A. Postma ${ }^{4,5}$. Tjasse D. Bruintjes ${ }^{6,7}$. Monique A. L. Bilderbeek-Beckers ${ }^{8}$. Patrick F. M. Dammeijer ${ }^{9}$. Stephanie Vanden Bossche ${ }^{10,11}$. Vincent Van Rompaey ${ }^{12}$. Philippe Lambin ${ }^{2}$. Marc van Hoof ${ }^{1}$. Raymond van de Berg' ${ }^{1}$}

1 Department of Otolaryngology and Head and Neck Surgery, Maastricht University Medical Center +, Maastricht, The Netherlands

2 The D-Lab, Department of Precision Medicine, GROW Research Institute for Oncology, Maastricht University, Maastricht, The Netherlands

3 Research and Development, Oncoradiomics SA, Liege, Belgium

4 Department of Radiology and Nuclear Medicine, Maastricht University Medical Center, Maastricht, The Netherlands

5 School for Mental Health and Sciences, Maastricht University, Maastricht, The Netherlands

6 Department of Otorhinolaryngology, Gelre Hospital, Apeldoorn, The Netherlands
7 Department of Otorhinolaryngology, Leiden University Medical Center, Leiden, The Netherlands

8 Department of Radiology, Viecuri Medical Center, Venlo, The Netherlands

9 Department of Otorhinolaryngology, Viecuri Medical Center, Venlo, The Netherlands

10 Department of Radiology, Antwerp University Hospital, Antwerp, Belgium

11 Department of Radiology, AZ St-Jan Brugge-Oostende, Bruges, Belgium

12 Department of Otorhinolaryngology and Head \& Neck Surgery, Antwerp University Hospital, Faculty of Medicine and Health Sciences, University of Antwerp, Antwerp, Belgium 